\title{
ANALYSIS OF STEERING GEAR UNDER THE REQUIREMENTS OF MODERN NAVIGATION
}

\author{
Gheorghe SAMOILESCU*, Dumitru IORGULESCU**, \\ Robert MITREA***, Laura D. CIZER* \\ *6"Mircea cel Bătrân" Naval Academy, Constanța, Romania \\ **Port Crewing, Portugal \\ ***CMC SHIPS Company \\ samoilescugheorghe@yahoo.com
}

\begin{abstract}
This paper presents aspects of the steering gear onboard a merchant ship by analyzing aft and bow systems based on automation and use of modern propulsion. The choice of the transverse propeller is based on several economic considerations (its price, consumption, efficiency, etc.), technical considerations (positioning, size, vibrations induced in the ship's hull), and maneuverability considerations (the ship's turning rate under the action of the propeller. Accordingly, the propulsion system can come in various sizes, power values, shapes of the tunnel, and can present fixed or variable pitch propellers. Depending on the maneuverability of the ship, the transverse propulsion is analyzed by taking into account two tests: the turning of the transverse propulsion system test in calm and windy weather, and the steering test.

The automation system is designed to control and monitor the on-board operational systems and equipment, and it encompasses a wide range of control, monitor and alarm. The integrated navigational equipment includes the following sub-systems: navigation consoles, ship handling consoles, dynamic positioning consoles, anchoring and deck operations consoles, and propulsion system control consoles.

The propulsion control system is especially dedicated to the propeller and thruster control system, resulting in a joint control system, and the cables are reduced in number since the communication lines are used in series. The mandatory condition for successfully solving the problem with the complex automation of naval installations and equipment is the construction of complex automatic control systems (ACS), consisting of: automated commands or remote controls, a system of collecting, processing and displaying information, as well as a system of control, fault detection and diagnosis.
\end{abstract}

Keywords: steering gear, modern propulsion, integrated navigational equipment, ACS

\section{Introduction}

The rudder is designed to achieve the necessary moment to action upon the rudder according to the standard orders received from the wheelhouse. The steering gear shall meet the requirements: the startup initiated by the steering wheel regardless of the position of the rudder; it shall be reversible in order to allow for the rudder to move in both sides; the rudder shall rotate in the same direction as the steering wheel; when the steering wheel stops, the rudder shall also stop and implicitly the rudder shall move; in extreme positions the steering wheel shall stop immediately; it shall be operated from several different places, it shall operate silently and have high safety in operation $[1,2]$.

The lateral force required for steering the ship to the desired trajectory is 
accomplished by the steering gear. Depending on how this force is obtained, there are: passive steering parts that produce the necessary lateral force for steering by using the water flow when the ship is underway, and active steering parts that produce the lateral force necessary for steering by transforming the mechanical energy they receive directly and specifically for steering purposes.

The aft steering gear is composed of two pumps with small axial pistons (port side and starboard side). They generate the necessary pressure for the hydraulic cylinders to operate. The subsystem containing the main pump with the electric drive motor and the associated hydraulic circuit elements is called the hydraulic power unit. Each hydraulic power unit consists of: the main pump and the auxiliary pump; the servo-valves (electrohydraulic and manual hydraulic ones); the bypass valve; the safety valve and purge valve; the electric drive motor.

For the steering gear (the rudder actuator) onboard the reference vessel, there are two linear hydraulic actuators (double acting hydraulic cylinders) acting on the tiller that is rigidly fixed to the rudder shaft. The transverse jet steering gear is represented by installations having a transverse tunnel located at the ends of the ship (or only in the bow) in which an axial propeller is mounted as shown in Figure 1.

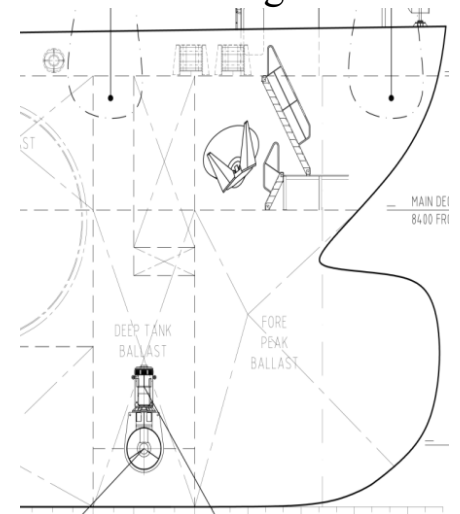

Figure 1: Transverse jet steering gear

The choice of the transverse propeller is based on several economic considerations (its price, consumption, and efficiency), technical considerations (positioning, size, vibrations induced to the ship's hull), maneuverability considerations (the ship's turning rate under the action of the propeller), and it may come in various sizes, power values, shapes of the tunnel, and may be equipped with fixed or variable pitch propellers, or may even have a variable number of propellers (1 or 2).

The bow thruster is used only for maneuvers in the port, mainly for unberthing operations while leaving and not underway, and it is effective only at anchor, or at very low speeds. The transverse propulsion system or "transverse propeller" shall be subjected to the following sea trials: gravity and steering tests $[3,4]$.

In calm weather, the sea trials begin with the ship in its leeway steady-state position as shown in Figures 2 and 3.

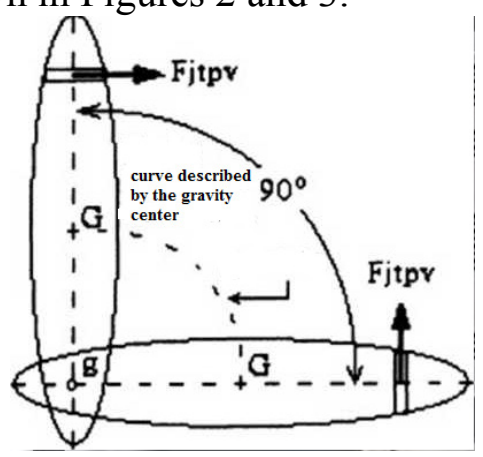

Figure 2: Gravity test of the transverse propulsion system in calm weather

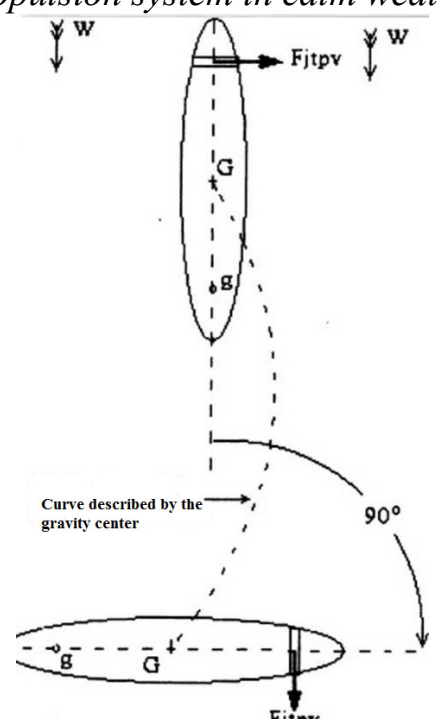

Figure 3: Gravity test of the transverse propeller steering gear in leeway 


\section{Integrated navigation system}

The operation of modern ships is closely related to the need to perform many tasks and adjustments both at anchor and underway $[5,6]$.

The manual operation requires a large number of operators, and practically some controls cannot be handled by them due to the fast speed of the process and to the high forces required to drive the control equipment. Some controls cannot be operated for a long time even by a welltrained operator, especially under ship operating conditions (roll, pitch, vibrations, noise, temperature, high ambient humidity). The problem of increasing the economic efficiency of the controls can only be solved by introducing complex automation. The manual operation (manual steering) is a method for steering that controls the rudder angle through the helm, and then the former follows the command given by the servo system. This control is effective when sailing in port, upon ship departure and arrival, or through channels. In the servo system, when the helm is rotated to the desired direction, the signal corresponding to the ordered angle is generated in the angle sensor connected to the helm. The signal goes to the rudder servo amplifier to produce a zero deviation between the steering angle and the current angle. For the steering gear (rudder drive) of the reference vessel, there are two double action hydraulic linear actuators (hydraulic cylinders) acting on tiller that is rigidly attached to the rudder shaft.

Based on the initial calculation data, a hydro-dynamic table calculation of the rudder was performed in Excel format, where the following parameters were calculated:

- Cqai - hydrodynamic coefficient of the moment relative to attack edge

- $\mathrm{Qi}-$ moment relative to rudder attack edge

- Cnai-hydrodynamic coefficient of normal force relative to attack edge

- Fnai-normal force acting on rudder blade

- CQRai-hydrodynamic coefficient

of the moment relative to rudder shaft

- QRai - moment relative to rudder shaft

The hydrodynamic calculation for the rudder was performed at rudder angle values ranging between $0^{\circ}$ and $35^{\circ}$, when the ship is going both ahead and astern. When going ahead, the service speed taken into consideration was $\mathrm{v}=15.5$ knots, and when going astern the speed $\mathrm{v}=5$ knots. After performing the rudder calculation, a maximum torque relative to the rudder blade was obtained: QR $\alpha \mathrm{i}=627.58 \mathrm{kNm}$ when going ahead, and QR $\alpha i=119.45$ $\mathrm{kNm}$ when going astern.

The proportional control function adjusts the amplitude of the rudder to the deviation signal, and the differential control function performs the so-called counter-rudder control while the integral control function automatically corrects the deviation to the set course when the deviation was generated by receiving the fixed external disturbances due to wind and current, etc. Wind and wave filtration functions are also included in the system. The fact that the rudder responds briefly to wind and wave disturbances becomes insignificant because the ship does not respond to this deviation, due to the large inertia of the ship's hull [7]. Complex automation allows the interconnection of all the technical means of the ship, ensures elevated technical and economic factors of the ordered processes, in all ship operation conditions, and eliminates the direct human participation in manning and operation $[8,9]$.

The mandatory condition for successfully solving the complex automation problem of the onboard installations and devices is the construction of complex automated systems which include: automatic controls or remote controls, an information collecting, processing and displaying system, as well as a control, fault detection and complex diagnostic system as shown in figure 4 . Depending on the automation volume, the 
following automation levels can be distinguished onboard the ship $[10,11]$ :

a) Zero level of automation. Controlled objects $(\mathrm{CO})$ together with direct manual control devices (DMCD) form the zerolevel automatic systems. For this level, the command is usually only in emergency situations.

b) The first level of automation. This level corresponds to automated drives (ADs) that together with $\mathrm{CO}$ form closed or open automated systems. It should be noted that in this case, the automation devices are usually not functionally linked to each other. In this case, the control is performed from local control stations (LCS) via simple control devices (buttons, switches, actuating levers), and $\mathrm{CO}$ information is received from various transducers. Objects controlled together with automated drives form the automated control objects. c) Second level of automation. The totally connected automated drives (ADs), control systems (CS), information processing systems (IPS) and control systems (CS) form the local complex automated system LCAS. This system corresponds to the second level of automation. The LCAS command is provided by the operator at the local command control boards (LCCs), which includes the control devices (CD) and the information display systems (IDS).

d) The third level of automation. The Automated Complex Ship System (ACSS) brings together the systems listed above with a control system (CS), one information processing system (IPS) and a control system (CS). The LCAS control is provided by the ship's central control station (CCS).

e) The fourth level of automation. This corresponds to the complex ship system (LCAS) which establishes the onboard overall control strategy, on the one hand, based on the information received from the LCAS, and on the other hand, from the higher rank ACS (for example from the ACS of the fleet).

f) The fifth level of automation. It allows for a considerable increase in the economic efficiency onboard ships, which not possible without the substantial and diverse help of the on-shore services. This is why complex automated systems at the fifth level are being built. These are ACSs corresponding to the respective production branch.

At the current automation stage, naval automation systems can be divided into several systems independent of each other, for example: automated power plant systems, automatic steering systems, automatic anchor gear, capstan and loading gear, each of them are an LCAS.

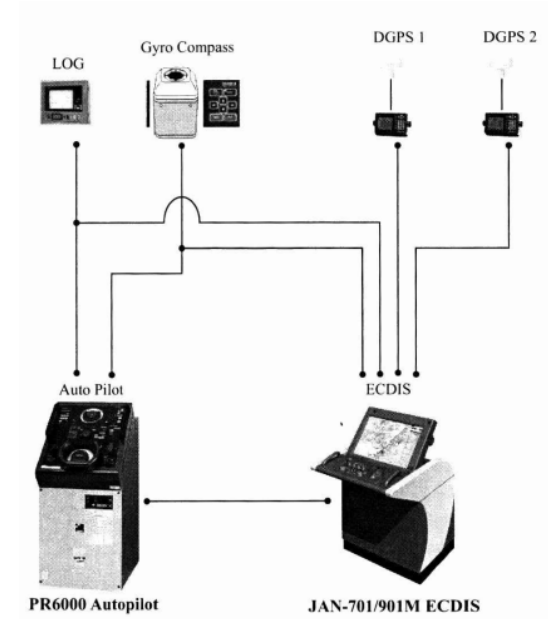

Figure 4:Linking diagram for Integrated Navigation System

\section{Automatic pilot control}

The PR6000 automatic pilot is a system that controls the rudder to maintain or change the ship's course. This system consists of a steering gear installed in the wheelhouse and equipment installed in the rudder room. These two units are interconnected with cables. In the event of a malfunction of the operating system, the steering functions can be performed by switching to another auxiliary system. This system controls the rudder rotations in such a way as to maintain the ship on its right course. There are two methods of controlling the rudder: (1) the follow up control (FU) which is used to control the rudder angle that causes the rudder to rotate automatically, (2) and the non follow up control in which the 
operator directly controls the rudder angle. In the follow-up control there are the following steering modes: the manual mode in which the helm indicates the rudder angle; the automatic mode in which the course is indicated by the course setting lever; the remote control mode in which the rudder angle or the course of the ship is indicated by another control system which is external to the control panel (in the remote control mode, manual remote control and equipment such as the Electronic Chart Display and Information System - ECDIS are mandatory). The automatic mode also includes the PID mode (proportional integral differention) that can manually control such steering features as weather adjustment etc., and the ADPT Adaptive Control (which is a steering control method in which the weather adjustment is automatically controlled in the course keeping mode and course changing mode during automatic steering. The main algorithm consists of the estimation unit - estimation of ship's hull dynamic characteristics - the adaptive Kalman filter unit and the optimal control unit, and the micro-processor is used to process input signals including external disturbances, and the optimal control can be performed without regard to sailing in calm or in rough sea [12].

This is the mode that can automatically control all these features.

The Non-follow up control (NFU) is the control method in which the steering angle is not quantitatively defined and the actual rudder angle has no feedback. Thus the steering needs to be done while seeing the rudder angle on the indicator. In the NFU (non follow up control) mode, the driving signal is sent directly to the control system of the steering gear. Since the signal is sent without passing through other processing circuits and servo-amplifiers, the rudder keeps moving as long as the port or starboard side button is pressed.

The self-adaptive operation mode (autosteering, adaptive control) is a method of steering in which adjustments (e.g. weather) are automatically controlled in course maintenance and course change modes during automated steering. The main algorithm is given by the estimation unit, the course maintenance controller, the course change controller, and the microprocessor is used to process input signals including external disturbances, and optimal control can be made without considering the navigation in calm or rough seas. The block automation block diagram in ADPT mode is divided into 3 units:

1. The Estimation Unit (estimates vessel characteristics). The ship's characteristics are changed by: its loading, speed, water depth and external disturbances such as wind, waves, etc. In conventional automated pilots that use PID control, the operator used to experience problems because it needed to change and adjust the PID parameters in order to get the adaptive direction each time the ship changed its characteristics. In the auto-ADPT mode of the system, the estimate algorithm is embedded to eliminate all the abovementioned problems and to always be able to get the optimal direction. This algorithm is based on the so-called NCT (New Control Technology). The NCT estimates the ship's characteristics as a mathematical model and uses the gained adjustment as its own control. In this system, this method is applied when changing the course in AUTO-ADPT mode.

2. The Course Maintenance Unit. When underway, the ship always receives external influences that influence the course of the ship. In general, in keeping the course in calm water, the ship handling system, including the automatic pilot, is mainly affected by reduced frequency components determined by the hull and the automatic pilot, while in rough sea it is affected by high frequency components induced by waves. The steering at high frequencies not only becomes ineffective, but also develops inertial resistance through forced and unnecessary steering of the rudder. In 
conventional automated pilots, linear filters and weather adjustments, such as nonlinear elements, have been developed to remove the high frequency components of the ship. In the new ADPT (NCT) mode, not only the ship's characteristics but also the disruptive elements are processed as a mathematical model.

3. The Course Change Unit. In the new ADPT (NCT) technology, the reference trajectory and the forward feed controller are used to automatically change the course. The trajectory is calculated taking into account the ship's characteristics and rudder characteristics.

AUTO - PID operation (autopilot, PID control) is a control method used by the PR6000 type pilot for automaticsteering. "P" indicates the proportional movement of the rudder adopting the steering angle proportional to the deflection angle, "I" indicates the integral movement of the rudder adopting the angle corresponding to the integrated value of the external disturbances, and "D" indicates the movement (differentiation, angular velocity). The deviation signal in automatic pilot mode is generated under the following conditions: the ship deviates from its established course while its current course has been changed to another course. In both cases, the set point signal and the set course signal go to the CPU (microprocessor) where it processes the PID operation. The computer calculates the rudder angle (proportional, differential, integral) based on the deviation signal, the set values of the PID (weather adjustment, angle adjustment rate, rudder angle adjustment, and specified return rate), and sends the angle signal to the control unit. The proportional control function adjusts the amplitude of the rudder (rudder angle) for the deviation signal and the differential control function performs the so-called anti-rudder control, and the integral control function (control I) automatically corrects the deviation to the set course when the deviation was generated by receiving external fixed wind and current disturbances, etc. Wind and wave filtration functions are also included in the system. The fact that the rudder responds briefly to the wind and wave disturbances becomes insignificant as the ship does not respond to this deviation, due to the large inertia of the ship's hull. Moreover, this type of deviation of the rudder, using the steering gear, is very inappropriate for its lifetime. The system includes the following subsystems: navigation and ship handling consoles, consoles for dynamic positioning (in ships where applicable), anchorage consoles and bridge operation consoles and consoles for controlling the propulsion system. They are built from standardized modules and components using control platforms where all systems work together through an integrated network where the information and control signals are securely interchanged so that the integrity of each system is secure.

\section{Propulsion Control System}

The Helicon X3 is the latest model of the successful Rolls Royce series (Helicon P \& $\mathrm{T}$ - Propeller \& Thruster) including the propeller and thruster control system. The control unit has an ergonomic and compact design with integrated push buttons for all functions including command transfer, knowledge of alarms, and on / off backup control. A system display located at each operator is of the touch screen type for propulsion units. The Helicon X3 uses the same components for all types of propulsion systems (propellers and thrusters) resulting in a unified control system, and its cables are reduced in number as serial communication lines are used. The automatic pilot system Aquapilot ND, developed by Rolls Royce, is an independent follow-up control system with a non-follow up back-up control system for a single azimuth, each thruster having an independent control system [13].

The system develops a precise, easy and safe direction of the thruster and can be an interface for the automatic ship system, 
autopilot and joysticks. The steering control system, alarms and rudder angle indication are provided for solenoid valves, and pumps and motors controlled by frequency converters. The system consists of a main control panel with emergency start / stop steering, NFU / FU mode selection, and small or large steering angle selection. The system is designed for the interface with the autopilot, joystick and voyage recorder.

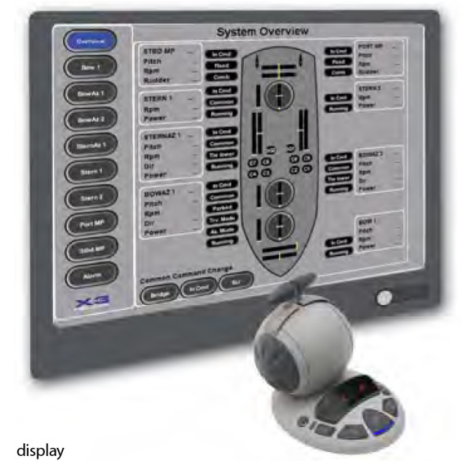

Figure 5: The Helicon X3 System

\section{Conclusions}

The steering gear is designed to keep the ship on its set course during navigation and to make course changes in accordance with the requirements of navigation by applying vertically rotating moments that act simultaneously to the axial propulsion force. The steering gear performs the rotation of the ship around the center of rotation during mooring and unberthing operations. The key features of the integrated navigation system are: multifunctional screens that allow the operator to choose which information to collect and present; critical and important information is shown simultaneously in order to ensure easy and safe operation; control consoles are placed ergonomically to allow the operator to work in sitting or standing positions with all the information clearly displayed on the "touch screen" screens; touch screen control systems are integrated into the support arms of the operator's seat.

The automation system stands for both integration and interfaces for any ship, system or equipment, and is developed by the Rolls Royce Company. The system is designed to control and monitor the ship's operational systems and equipment, and includes a wide range of controls, monitors and alarms. Multifunctional screens allow the operator to choose between the system control and the information to be presented. The user interface is adapted to the ship and provides access to its basic systems. The main functions of the integrated automated system are: alarms and monitoring, machinery control, cargo control, cargo monitoring, and operational control of the ship.

\section{References}

[1] Magnusson.T, Meeting- A boats behaviour and important parameters, 2017

[2] TMQ International- How does an autopilot work [online]. Available at: http:// tmqeurope. com/information/how-does-an-autopilot-work

[3] www.Standard ship test and inspection plan, procedures and data base, U. S. Departament of the Navy Carderock Division, Naval Surface Warface Center,1999-2017

[4] Procedure of testing steering gears on ship, 2017 https://www. marineinsight.com/guidelines/procedure-of-testing-steering-gears-on-ship/

[5] Integrated navigation system-www.pfri.uniri.hr/-bopri/documents/31-ME-tal.pdf

[6] Integrated navigation system - www. raytheon-anschuetzcom/products solutions/integrated-bridge-system/synapsis-ins-ibs/

[7] Samoilă C Instalatii navale-Elemente de calcul numeri şi proiectare, 2017

[8] Lundberg Eric Karlsson. P-Enhanced auto pilot for marine applications with adaptive speed control, Department of Electrical Engineering, Chalmers University of Technology, Gothenburg, Sweden, 2017 
[9] Frisk, D, A Chalmers University of Technology Master's thesis temple for LATEX Unpublished, 2016

[10] Marine Control Systems- Integrated Systems for all aspects of ships-www. grighthubengineering.com-marine-engines-machinery-73565-ship-control-systems

[11] Marine-Siemens-w3.siemens.no/home/no/no/sector/industry/marine/pages/integratedautomation-systems.aspx

[12] www.lemarsg.com/images/Pdf/autopilot-tokimec-pr6000-manual.pdf,2001

[13] www. Rolls Royce Marine A.S, Propulsion-Ulsteinvik-Lifting of propulsion unit. Instalation onboard-Aquamaster, Azimut Thrusters 